\title{
Pragmatic model of patient satisfaction in general practice: progress towards a theory
}

\author{
Richard Baker
}

\begin{abstract}
A major problem in the measurement of patient satisfaction is the lack of an adequate theory to explain the meaning of satisfaction, and hence how it should be measured and how the findings are interpreted. Because of the lack of a fully developed theory, when developing patient satisfaction questionnaires for use in general practice, a simple model was used. This model was pragmatic in that it linked together empirical evidence about patient satisfaction without recourse to more general social or psychological theory of behaviour, other than to define satisfaction as an attitude. Several studies with the questionnaires confirm in general the components of the model. However, the importance of personal care had not been sufficiently emphasised, and therefore the model has been revised. It can now serve as a basis for future research into patient satisfaction, in particular as a stimulus for investigating the links between components of the model and underlying psychological or other behavioural theories. (Quality in Health Care 1997;6:201-204)
\end{abstract}

Keywords: patient satisfaction; theory; general practice

Eli Lilly National Clinical Audit Centre, Department of General Practice and Primary Health Care, University of Leicester Richard Baker, director

Correspondence to: Dr Richard Baker, Eli Lilly National Clinical Audit Centre, Department of General Practice and Primary Health Care, University of Leicester, Leicester General Hospital, Gwendolen Road, Leicester LE5 4PW, UK.

Accepted for publication 1 October 1997

\section{Introduction}

It is easy to devise a simple questionnaire on patient satisfaction, and when asked, most patients in general practice will obligingly complete it. Yet when the responses are analysed, the findings are often singularly unhelpful. According to the questionnaire, most patients are satisfied, but in most practices receptionists and other staff often receive grumbles from patients. It would seem that the questionnaire has failed to elicit patients' true level of satisfaction. How can measurement of patient satisfaction be improved?

A particular problem has been the lack of a theory to explain the meaning of patient satisfaction. ${ }^{1-4}$ In the absence of a theory it is difficult to decide how to measure satisfaction or how the findings of surveys should be interpreted. The Concise Oxford Dictionary defines a theory as being a "supposition explain- ing something, especially one based on principles independent of the phenomena". A theory can be used to explain observations and predict consequences, and can be tested in empirical research. For example, the theory of smoking and lung cancer asserts that certain constituents of tobacco smoke have an effect on the function and structure of susceptible cells, which then become malignant. The theory also suggests what the consequences are likely to be for the patients concerned, and what treatments might be helpful, all matters that can be investigated in research studies. Thus, theories can be regarded as ideas which help to explain observations and suggest new hypotheses.

Several approaches have been taken in the past to develop a theory of patient satisfaction. Linder-Pelz linked psychological and social theories of attitudes ${ }^{5}$ but in a small study in primary care was able to explain only a limited proportion of observed differences in satisfaction, the most important factor being patient expectations. ${ }^{6}$ Other studies have confirmed that expectations play some part. ${ }^{7}$ However, expectations have been classified as cognitive whereas satisfaction is an affective state, and although attempts have been made to link them a complete theory is lacking. ${ }^{8}$ Lay ideas, ${ }^{9}$ discrepancy theory, and the structural relation between patient and healthcare agents have also been proposed for understanding satisfaction, but have yet to be fully evaluated. ${ }^{10}$ In North America, marketing theories are now being investigated, ${ }^{11-13}$ but the extent to which these can be applied in a less consumerist general practice service in the United Kingdom is unclear.

In the absence of a theory, I developed a questionnaire with a model to measure patient satisfaction with practices ${ }^{14}$ and consultations. ${ }^{15}$ A dictionary definition of a model is "a simplified description of a system". Thus, in contrast to a theory, a model merely depicts the relation between a set of variables, rather than explains them on the basis of underlying principles. In this paper, the model is described and subjected to critical review in the light of findings from the questionnaires.

The questionnaires are the surgery and consultation satisfaction questionnaires. They 
were developed with psychometric methods from an original list of 139 questions identified from published surveys and comments made by patients to a primary healthcare team, supplemented by inclusion of open questions on the first pilot versions. The surgery questionnaire considers six components of satisfaction-general satisfaction, accessibility, availability, continuity, medical care, and premises. The consultation questionnaire considers four components of satisfaction with consultations-general satisfaction, professional care, depth of relations, and perceived time. The final versions have been tested for reliability and validity, ${ }^{16}$ and have been validated in comparison with qualitative methods. ${ }^{17}$ The consultation questionnaire has been compared with the medical interview satisfaction scale, ${ }^{18}$ was used in studies of videotaped consultations, ${ }^{19}$ and was used to assess the effects on satisfaction of detecting emotional disturbance in patients. ${ }^{20} \mathrm{~A}$ modified version has now been developed to assess patients' satisfaction with consultations with community nurses. ${ }^{21}$

\section{Pragmatic model}

The model was pragmatic in that it linked together available empirical evidence about patient satisfaction without recourse to more general social or psychological theories of behaviour, other than to define satisfaction as an attitude (fig 1). Firstly, that attitude was defined as an evaluative judgement (or reaction to) care received. ${ }^{5}$ As with other attitudes, it is learned from experience, is relatively enduring in comparison with emotional states - such as anger or amusement-and exerts some influence on behaviour. ${ }^{22}$

Secondly, satisfaction is regarded in the model as a continuous rather than dichotomous variable (after Ware ${ }^{23}$ ). Thirdly, it is multidimensional, different elements of care each causing differences in satisfaction. ${ }^{24} \mathrm{~A}$ patient may be satisfied with one element of caresuch as the appointment system-but dissatisfied with another such as the clinical examination. Thus, a measure of overall satisfaction will be a summary of the competing evaluations of the patient and may not be sensitive to differences in levels of satisfaction with individual elements of care. Furthermore, in different clinical settings different elements of care may be more or less important. For example, in general practice continuity may be viewed by patients as particularly important,

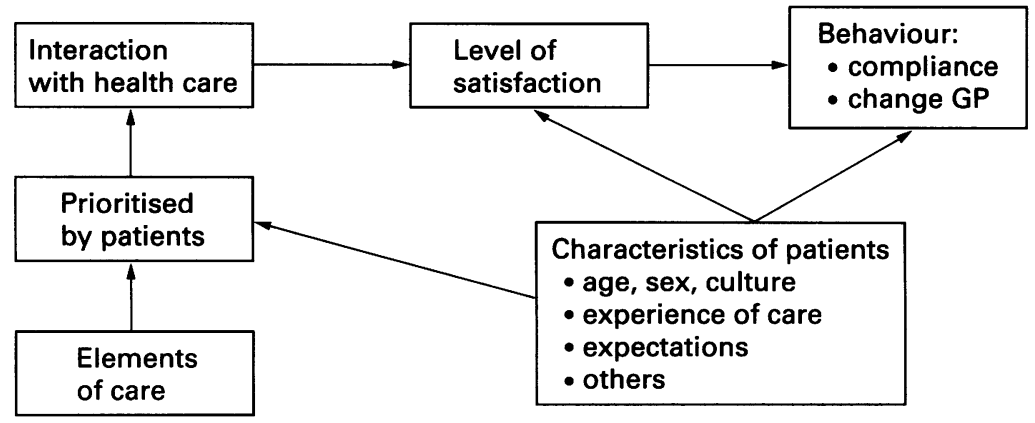

Figure 1 Initial model of patient satisfaction in general practice.
A summary of the principal findings of studies that used surgery (SSQ) and consultation satisfaction questionnaires(CSQ)

\section{SATISFACTION AND CONTINUITY}

Study in two general practices involving 711 patients

Continuity was calculated as the percentage of past 12 consultations that were with the patient's usual doctor. Lower levels of continuity were associated with lower levels of satisfaction as measured by SSQ and CSQ (at $100 \%$ continuity, SSQ general satisfaction was 81.5 and CSQ general satisfaction 78.3 , at $25 \%$ continuity satisfaction scores were 67.3 and 71.3 respectively).

\section{SATISFACTION WITH PRACTICES}

The SSQ was completed by 16015 patients attending 89 practices. There were wide variations in levels of satisfaction between practices, and multiple regression showed that the practice characteristics associated with satisfaction were total list size (satisfaction falls as list size increases), presence of a personal list (satisfaction increases), and being a training practice (satisfaction falls).

\section{SATISFACTION WITH CONSULTATIONS}

The CSQ was given to 7273 patients attending 126 general practitioners. Multiple regression showed that the practice characteristics associated with satisfaction with consultations were total list size (satisfaction falls as list size increases), presence of a personal list (satisfaction increases), being a training practice (satisfaction falls), and the number of patients booked in the appointment system per hour (an increase in the number of patients booked was associated with falls in satisfaction with the duration of consultations).

but in a surgical outpatient clinic the provision of comprehensive information about treatment choices may be more important. It follows that measures of satisfaction should assess all relevant elements of care and be designed for particular clinical settings.

Fourthly, patients' characteristics may influence their attitudes towards care, and also the importance they assign to different elements of care. In the model, patients' characteristics are shown as influencing the priorities they assign to different elements of care and to their attitude or level of satisfaction after an interaction with the healthcare system. Some patients may allocate the highest priority to continuity, others may prefer readily available appointments. Patients' characteristics that may be influencial include age and $\operatorname{sex}^{24}$ past experience of care, ${ }^{12}$ expectations, ${ }^{10}$ health, ${ }^{25}$ cultural factors, mood, and others. Finally, satisfaction can influence patients' future behaviour such as compliance with advice ${ }^{26}$ or whether they change doctors. ${ }^{27}$ 


\section{Assessment of the model}

The model has been assessed with the questionnaires in several studies (box). These include validation studies ${ }^{14}{ }^{16}$ and investigation of the characteristics of patients, practices, and general practitioners, which influence satisfaction. ${ }^{28}{ }^{29}$ These studies do not directly test whether satisfaction is an attitude, although they do indicate that the level of satisfaction is relatively stable over a two week interval and is associated with the behaviour of changing general practitioners. ${ }^{16}$ The findings were also compatible with satisfaction being a continuous variable, as the responses of patients to questions were distributed across all response choices and levels of satisfaction varied widely depending on the practices ${ }^{28}$ and general practitioners ${ }^{17}{ }^{29}$ that the patients attended. For example, in some practices most patients expressed dissatisfaction with the availability and continuity of care.

The multidimensional nature of satisfaction was confirmed by principal components analysis which identified several elements of care that patients evaluated differently. Furthermore, each component of satisfaction was shown to have moderate correlation with general satisfaction, indicating that they were related to, and not simply manifestations of, general satisfaction. ${ }^{14}{ }^{15}$ The findings also tend to support the model's proposition that some elements of care are viewed as more important than others. The range of scores for availability and continuity were wider, and the mean scores lower, than for other components of the surgery satisfaction questionnaire, suggesting that patients were more critical of these components than they were of premises or medical care. ${ }^{28}$

The findings also supported the fourth feature of the model, that patient characteristics may influence satisfaction. The studies did not investigate expectations, health status, or past experiences of health care other than continuity. However, there were differences in satisfaction depending on patient age and sex. For example, satisfaction with the depth of relation fell with increasing age of women patients, and also fell as the proportion of men consulting increased. ${ }^{29}$ As patients increased in age, they were more likely to express satisfaction with the availability of appointments or medical care, provided the practice operated a personal list system. ${ }^{28}$ Only the basic characteristics of age and sex were investigated, and it is conceivable that other characteristics would influence

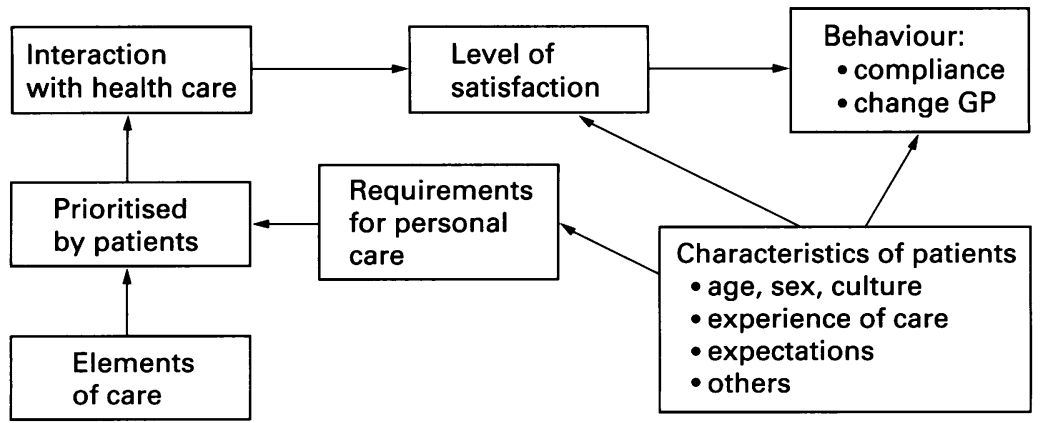

Figure 2 Revised model of patient satisfaction in general practice. satisfaction-such as employment, expectations, or health. ${ }^{725}$

The model also argues that satisfaction influences some subsequent behaviours of patients. The only behaviour investigated was that of changing general practitioner without a change of home address. Patients who changed had lower levels of satisfaction with their previous general practitioner. ${ }^{16}$

The model suggests that some elements of care may be more important to patients than others, and that in different settings their relative importance may vary. In particular, higher levels of continuity were shown to be related to higher satisfaction. ${ }^{16}$ Scores for the surgery satisfaction questionnaire were higher if practices were smaller, operated personal list systems, and were not training practices. ${ }^{28}$ In general, scores for the consultation satisfaction questionnaire were also higher in smaller practices, those that had personal list systems, and were not training practices. ${ }^{29}$ Patients report greater availability in smaller practices. ${ }^{30}$ Personal list systems can be associated with higher levels of continuity, ${ }^{31}$ and in training practices the regular departure of one doctor and arrival of a new one can, for some patients, disrupt continuity. Thus, personal lists, continuity, practice size, and the regular replacement of doctors in training practices are factors that may limit the opportunity for the relation between patient and doctor to develop to the point at which the patient feels that care is personal.

The extent to which care is perceived as personal may be influenced by several factors, including continuity or the relation in consultations, and further research is needed. Furthermore, although most patients regard personal care as important, some may not. Infrequent consulters, people who change address often, or some working people may regard convenient availability as more important. Nevertheless, for many patients it seems to be an underlying element of care that has a pervasive influence on satisfaction. The importance attached to personal care had not been a feature of the pragmatic model, and it was revised to take this into account (fig 2).

\section{Implications of the model for satisfaction} theory, measurement, and research Most questionnaires on patients' satisfaction are developed without reference to relevant theories or models. However, the use of a model has important advantages. It indicates how satisfaction should be measured and helps to explain the practical significance of the findings. Satisfaction was regarded as an attitude, a psychological concept which has recently been defined as "a psychological tendency that is expressed by evaluating a particular entity with some degree of favour or disfavour". ${ }^{32}$ One advantage of conceptualising satisfaction as an attitude is that established methods of measuring attitudes can be used. Also, links can be drawn between the model and underlying psychological theory. General theories of attitudes and associated empirical studies may improve our understanding of patients' attitudes towards the care they receive. However, although 
psychological theories may be helpful in explaining satisfaction, further research is needed to determine which theories are applicable. For example, the ability of the theory of reasoned action $^{33}$ to explain satisfaction has only been investigated in one small study which found little support for it, although there was limited support for discrepancy theory. ${ }^{6}$ Other theories that might help to explain patient satisfaction include information integration and information processing theories, ${ }^{32}$ but these have not yet been considered in research studies.

As well as defining patient satisfaction as an attitude, the pragmatic model indicates that various patient and health service variables influence satisfaction, and raises questions for future research. These include the process by which patients assign priorities to different elements of care, the relation between levels of satisfaction and subsequent health related behaviour, the role of cultural values, and the extent to which patients' attitudes to health care can be viewed as consumerist. Personal care is a concept that requires exploration and definition, and its role in influencing satisfaction in different cultures should be explored. Studies are needed to determine whether other salient beliefs are equal or more important to different patients in different healthcare settings.

The pragmatic model also has implications for methods of measuring satisfaction. One factor that should be taken into account is the multidimensional nature of satisfaction. If levels of satisfaction with only a few elements of care are measured, mistaken conclusions might be drawn. For example, in the evaluation of a new clinic in a general practice, patient satisfaction with the clinic might be found to be high, and the value of the clinic assumed to be proved. However, there may have been a decline in satisfaction with continuity or the availability of appointments which would have gone unnoticed. Reliance on measurement of general satisfaction alone is not an adequate alternative because general satisfaction is influenced by so many elements of care that discrimination between different levels of satisfaction is likely to be poor.

The pragmatic model has served a useful function in guiding the development and use of two measures of satisfaction, and may be of use to others. Its most important implication is that future studies of patient satisfaction should draw on models such as that described in this paper, and when possible test the relations between such models and underlying theories. It will only be through systematic investigation of the links between theory and empirical observation that understanding of patients' attitudes will be improved and will come to play a full part in the planning and delivery of health care.

I thank all those who have helped me develop and employ the satisfaction questionnaires, in particular Dr Michael Whitfield, satisfaction questionnaires, in particular Dr Michael Whitfield, Dr Havid Armstrong, and Professor RC Fraser. I am grateful to on an early draft of this paper. The Eli Lilly National Clinical
Audit Centre is funded jointly by Lilly Industries and Leicestershire Health Authority, and has complete academic autonomy.

1 Wensing $M$, Grol R, Smits A. Quality judgements by patients on general practice care: a literature analysis. Soc Sci Med 1994;38:45-53.

2 van Campen C, Friele RD, Kerssens JJ. Methods for assessing patient satisfaction with primary care. Review and annotated
bibliography. Utrecht: Institute of Primary Care, 1992. bibliography. Utrecht: In

3 Lewis JR. Patient views on quality in general practice: literature review. Soc Sci Med 1994;39:655-70.

4 Locker D, Dunt D. Theoretical and methodological issues in sociological studies of consumer satisfaction with medical care. Soc Sci Med 1978;12:283-92.

5 Linder-Pelz S. Toward a theory of patient satisfaction. Soc Sci Med 1982;16:577-82.

6 Linder-Pelz S. Social psychological determinants of patient satisfaction: a test of five hypotheses. Soc Sci Med 1982;16: 583-9.

7 Williams S, Weinman J, Dale J, Newman S. Patient expectations: what do primary care patients want from the GP and how far does meeting expectations affect patient satisfaction? Family Practice 1995;12:193-201.

8 Thompson AGH, Sunol R. Expectations as determinants of patient satisfaction: concepts, theory and evidence. Int $\mathcal{f}$ Qual Health Care 1995;7:127-41.

9 Calnan M. Towards a conceptual framework of lay evaluation of health care. Soc Sci Med 1988;27:927-33.

10 Carr-Hill RA. The measurement of patient satisfaction. $f$ Public Health Med 1992;14:236-49.

11 Ross CK, Frommelt G, Haxelwood L, et al. The role of expectations in patient satisfaction with medical care. fournal of Health Care Marketing 1987;7:16-26.

12 John J. Patient satisfaction: the impact of past experience. fournal of Health Care Marketing 1992;12:56-64.

13 Aharony L, Strasser S. Patient satisfaction: what we know about and what we still need to explore. Medical Care Review 1993;50:49-79.

14 Baker $R$. The reliability and criterion validity of a measure of patients' satisfaction with their general practice. Fam Pract 1991;8:171-7

15 Baker R. Development of a questionnaire to assess patients' satisfaction with consultations in general practice. $\mathrm{Br} \mathcal{F} \mathrm{Gen}$ Pract 1990;40:487-90.

16 Baker $R$, Whitfield $M$. Measuring patient satisfaction: a test of construct validity. Ouality in Health Care 1992;1:104-9.

17 Lewis JR, Williamson V. Examining patient perceptions of quality care in general practice: comparison of quantitative and qualitative methods. Br F Gen Pract 1995;45:249-53.

18 Kinnersley P, Stott N, Peters T, Harvey I, Hackett P. A comparison of methods for measuring patient satisfaction with consultations in primary care. Family Practice 1996;13:4151.

19 Campbell LM, Sullivan F, Murray TS. Videotaping of general practice consultations: effect on patient satisfaction. BMf 1995;311:236.

20 Wilson PMJ, Sullivan F, Hussain S, Smith GD. Examination of the effects of emotional disturbance and its detection on general practice patients' satisfaction with the consultation. Br f Gen Pract 1995;45:304-8.

21 Poulton BC. Use of the consultation satisfaction questionnaire to examine patients' satisfaction with general practitioners and community nurses: reliability, replicability and discriminant validity. Br $\mathcal{F}$ Gen Pract 1996;46:2631 .

22 O'Keefe DJ. Persuasion. Theory and research. Newbury Park: Sage, 1990.

23 Ware JE. How to survey patient satisfaction. Drug intelligence and clinical pharmacy. 1981;15:892-9.

24 Hall JA, Dornan MC. Patient sociodemographic characteristics as predictors of satisfaction with medical care: a meta-analysis. Soc Sci Med 1990;30:811-8.

25 Hopton JL, Howie JGR, Porter AMD. The need for another look at the patient in general practice satisfaction surveys. Fam Pract 1993;10:82-7.

26 Kincey J, Bradshaw P, Ley P. Patients' satisfaction and reported acceptance of advice. Fournal of the Royal College of General Practitioners 1975;25:558-66.

27 Marquis MS, Davies AR, Ware JE. Patient satisfaction and change in medical care provider: a longitudinal study. Med Care 1983;21:821-9.

28 Baker R, Streatfield J. What type of general practice do patients prefer? Exploration of practice characteristics influencing patient satisfaction. $\mathrm{Br} \mathcal{F}$ Gen Pract 1995;45: 654-9.

29 Baker R. Characteristics of practices, general practitioners and patients related to levels of patients' satisfaction with consultations. Brf Gen Pract 1996;46:601-5.

30 Campbell JL. The reported availability of general practitioners and the influence of practice list size. $\mathrm{Br} \mathcal{f}$ Gen Pract 1996;46:465-8.

31 Freeman GK, Richards SC. How much personal care in four group practices? BMF 1990;301:1028-30.

32 Eagly AH, Chaiken S. The psychology of attitudes. Orlando: Harcourt Brace, 1993.

33 Fishbein $M$, Ajzen I. Predicting and understanding consumer behaviour: attitude-behaviour correspondence. In: Ajzen I, Fishbein M, eds. Understanding attitudes and predicting social behaviour. Englewood Cliffs, NJ: Prenticepredicting social beha 